\title{
Exigências energéticas para mantença e para o crescimento de frangos de corte $^{1}$
}

\author{
Flavio Alves Longo 2, 3 , Nilva Kazue Sakomura², Carlos Boa-Viagem Rabello2, 4 , Adriana \\ Nogueira Figueiredo ${ }^{2}$, João Batista Kochenborger Fernandes ${ }^{5}$
}

\footnotetext{
1 Parte da Dissertação de Mestrado do primeiro autor.

2 Departamento de Zootecnia, UNESP, Jaboticabal, SP.

${ }^{3}$ BTECH, São Paulo, SP.

${ }^{4}$ Departamento de Zootecnia, UFRPE, Recife, PE.

5 CAUNESP - UNESP, Jaboticabal, SP.
}

RESUMO - O trabalho foi realizado com o objetivo de determinar, pelo método fatorial, as exigências de energia metabolizável (EM) de frangos de corte. Foi conduzido um ensaio em câmaras climáticas mantidas às temperaturas de 13,23 e $32^{\circ} \mathrm{C}\left( \pm 2^{\circ} \mathrm{C}\right)$ para se verificar o efeito da temperatura sobre as exigências de energia metabolizável para mantença utilizandose a técnica do abate comparativo. As exigências de energia metabolizável para ganho de peso foram determinadas com base no teor de energia corporal e na eficiência energética de utilização da EM. As exigências de mantença foram de 159,36; 116,17 e $128,66 \mathrm{kcal} / \mathrm{kg}^{0,75} / \mathrm{dia}$ para 13,23 e $32^{\circ} \mathrm{C}$, respectivamente, verificando-se efeito quadrático da temperatura sobre as exigências de mantença $\left(E M m=300,14-14,61 . T+0,2876 . T^{2}, r^{2}=0,90\right)$. As exigências de EM para ganho de peso corporal foram de $3,72 \mathrm{kcal} / \mathrm{g}$ para machos e $3,98 \mathrm{kcal} / \mathrm{g}$ para fêmeas de 1 a 21 dias; 4,21 para machos e 3,93 para fêmeas de 22 a 42 dias; e 4,51 para machos e 7,04 para fêmeas de 43 a 56 dias. Considerando-se as exigências determinadas, foram elaborados modelos de predição das exigências diárias de energia para frangos de corte, nos quais foram considerados o peso corporal, a temperatura ambiente (para estimativa das exigências de mantença) e o ganho de peso (para cálculo das exigências de ganho). Com base nas comparações das exigências determinadas pelos modelos e nas recomendações do manual da linhagem, conclui-se que os modelos elaborados predizem as exigências energéticas dos frangos de corte.

Palavras-chave: exigências de energia, frangos de corte, método fatorial, temperatura ambiente

\section{Metabolizable energy requirements for maintenance and growth of broilers}

\begin{abstract}
This work was conducted to determine the energy requirements for broilers using the factorial method. The metabolizable energy (ME) requirements for maintenance (MEm) were determined at 13,23 and $32^{\circ} \mathrm{C}( \pm 2 \mathrm{C})$ by the comparative slaughter technique. ME requirements for weight gain were estimated considering the net efficiency of energy utilization for growth and body energy content. The energy requirements for maintenance were of 159.36, 116.17, and 128.66 $\mathrm{kcal} / \mathrm{kg}^{0.75} / \mathrm{day}$ at 13,23 , and $32^{\circ} \mathrm{C}$, respectively, and quadratic effect of temperature $(\mathrm{T})$ on the maintenance requirement $\left(\mathrm{MEm}=300.14-14.61 \mathrm{~T}+0.2876 \mathrm{~T}^{2}, \mathrm{r}^{2}=0.90\right)$ was detected. Metabolizable energy requirements for weight gain for males and females were of 3.72 and $3.98 ; 4.21$ and $3.93 ; 4.51$ and $7.04 \mathrm{kcal} / \mathrm{g}$, for the periods from 1 to 21,22 to 42 and 43 to 56 days of age, respectively. Considering the requirements, prediction models were designed for ME requirements using adjustments for body weight, daily weight gain and temperature. The models predicted the ME requirements for broilers as redult of from breed manual recommendations and the studied models.
\end{abstract}

Key Words: broilers, energy requirements, factorial method, temperature

\section{Introdução}

Atualmente, as pesquisas, tanto em genética como nutrição e manejo, visam à redução dos custos de produção sem prejudicar o desempenho animal. Por isso, é importante explorar o metabolismo energético das aves, estudando todos os fatores que o afetam, e o aproveitamento dos demais nutrientes da dieta, facilitando a manipulação das rações para melhorar as características de carcaça, favorecer a deposição de proteína e diminuir o acúmulo de gordura.

Nesse contexto, torna-se essencial a determinação das exigências energéticas, das eficiências de aproveitamento da energia e de deposição de proteína e gordura (Scott et al., 1982).

As exigências energéticas podem ser estimadas por dois métodos: o empírico, baseado no desempenho das aves em resposta a variados níveis de ingestão de 
energia, e o fatorial, baseado na exigência energética da ave para satisfazer a manutenção dos processos vitais, do ganho de peso e/ou crescimento (Hurwitz et al., 1983).

O método fatorial constitui a base para os diversos modelos matemáticos desenvolvidos para estimativas das exigências nutricionais e pode ser expresso pelo modelo: $\mathrm{CN}=\mathrm{Nm}+\mathrm{Ntm}+\mathrm{Ng}$, em que $\mathrm{CN}$ é o consumo do nutriente e $\mathrm{Nm}, \mathrm{Ntm}$ e $\mathrm{Ng}$, as demandas de nutrientes para manutenção, retenção de tecido magro e retenção de gordura corporal, respectivamente. Nm é dependente do peso e da composição corporal, Ntm é destinado à maximização, mas é limitado pelo potencial genético de deposição de tecido magro, e o CN e Ng sofrem influências ambientais e genéticas (Emmans, 1986). O frango de corte moderno possui maiores rendimentos de carne e peito e mais alta deposição de gordura, que estão relacionados à genética. Entretanto, o desempenho e a composição da carcaça podem sofrer influência da nutrição, do sexo e das condições ambientais (Albino et al., 2000).

Os fatores normalmente estudados que mais influenciam as exigências de energia para aves são o peso corporal, a taxa de crescimento, a temperatura, o grau de atividade física e o grau de empenamento. No entanto, segundo Zhang \& Coon (1994), existem outros fatores menos estudados como: umidade, movimento do ar e densidade das aves.

Com a utilização de modelos de predição, é possível determinar as exigências energéticas considerando as diferenças entre linhagens, o estágio de produção, as regiões e épocas do ano, permitindo a elaboração mais precisa de tabelas de exigências de energia para aves (Sakomura et al., 1993).

Este trabalho foi realizado com os objetivos de estudar o metabolismo energético de frangos de corte, pela determinação das exigências energéticas para mantença e ganho de peso, elaborar modelos de predição das exigências de energia para machos e fêmeas nas diferentes fases de criação e verificar suas aplicações em simulações matemáticas.

\section{Material e Métodos}

Os experimentos foram desenvolvidos no aviário experimental do Departamento de Zootecnia da Faculdade de Ciências Agrárias e Veterinárias, UNESP, Jaboticabal.

Para determinação das exigências de energia para mantença e do efeito da temperatura e do nível de alimentação sobre esses valores, foram conduzidos ensaios em três câmaras climatizadas com temperaturas de 13,23 e $32 \pm 2^{\circ} \mathrm{C}$. Quatrocentos e oitenta frangos de corte machos da marca comercial AgRoss 308 com 14 dias de idade permaneceram nas câmaras, sobre piso de cama de maravalha durante 35 dias (sete para adaptação às temperaturas e 28 para coleta de dados). Em cada temperatura, as aves foram distribuídas em quatro tratamentos, com quatro repetições de 10 aves. Os tratamentos consistiram de quatro níveis de alimentação (ad libitum, 70, 50 e 30\% do consumo ad libitum).

As dietas experimentais foram formuladas segundo recomendações para a linhagem (Agross, s.d.), atendendo às necessidades nutricionais das aves para cada fase.

Simultaneamente, ensaios de metabolismo foram realizados utilizando-se o método de coleta total de excretas, para determinação da energia metabolizável aparente corrigida pelo balanço de nitrogênio (EMAn) das rações experimentais utilizadas em cada fase de criação. Um grupo de aves da marca comercial AgRoss 308 (machos e fêmeas) foi alojado em gaiolas distribuídas pelos quatro níveis de alimentação (ad libitum, 70, 50 e $30 \%$ do consumo ad libitum) e três repetições para cada sexo.

Para determinação das exigências de energia de mantença, o alimento foi fornecido diariamente em quantidade ajustada com base no consumo do dia anterior do tratamento ad libitum, permitindo o controle da ingestão de energia metabolizável (EM), considerando-se os valores de EMAn das dietas determinados nos ensaios de metabolismo.

Foram realizados dois abates referenciais: um inicial, após o final do período de adaptação das aves, aos 21 dias de idade, e outro final, aos 49 dias de idade. Após o abate das aves, as penas foram removidas e as carcaças foram congeladas e moídas integralmente, para obtenção de uma amostra representativa. Em seguida, amostras de penas e carcaças foram secas em estufa, moídas novamente, sendo determinados os teores de matéria seca e energia bruta.

A partir dos teores médios inicial e final de energia na carcaça e nas penas, foi determinado o conteúdo de energia corporal total ao início e final do período experimental e, por diferença, estimou-se a energia corporal retida no período.

As exigências de EMm foram calculadas pela seguinte fórmula: $E M m=I E M-(E R / k)$, em que IEM = ingestão de energia metabolizável $\left(\mathrm{kcal} / \mathrm{kg}^{0,75} / \mathrm{dia}\right), \mathrm{ER}=$ energia retida corporal ( $\left.\mathrm{kcal} / \mathrm{kg}^{0,75} / \mathrm{dia}\right)$ e k = eficiência de utilização da energia para retenção corporal, determinada em experimento realizado por Longo (2000) nas mesmas condições de temperatura adotadas neste estudo - 61, 58 e $66 \%$ nas temperaturas de 13,23 e $32^{\circ} \mathrm{C}$, respectivamente.

$\mathrm{O}$ efeito da temperatura sobre a exigência de EM para mantença foi determinado pela regressão dos valores de EM para mantença, estimados de acordo com a temperatura.

Para determinação das exigências de energia para ganho de peso, foi conduzido um ensaio em temperatura ambiente, utilizando-se 180 frangos de corte machos e 180 fêmeas AgRoss 308 com um dia de idade, distribuídos em 
oito grupos de 45 aves (quatro grupos para cada sexo). Semanalmente, foram registrados a ingestão de alimento, o peso corporal, o ganho de peso e o teor de energia na carcaça, por meio de abates semanais durante o período de 7 a 56 dias de idade.

Pela regressão do teor de energia na carcaça em função do peso corporal, determinou-se a exigência de energia líquida para ganho de peso, a qual, dividida pela eficiência de utilização da EM da dieta, forneceu a exigência de EM para ganho de peso.

A eficiência de utilização da EM da dieta para o ganho de peso (59\%) foi considerada como o coeficiente de regressão da equação da energia retida, de acordo com a EM ingerida, estabelecida em ensaio realizado por Longo (2000) para determinação da exigência de EM para mantença em ambiente termoneutro $\left(23^{\circ} \mathrm{C}\right)$.

As análises de regressões lineares, seus coeficientes de determinação e sua significância foram obtidos por meio do programa Statistica (1996).

Considerando-se as exigências de mantença e ganho e as eficiências determinadas, foram elaborados modelos de predição das exigências diárias de energia metabolizável para frangos de corte machos e fêmeas de 1 a 21, 22 a 42 e 43 a 56 dias de idade.

Os objetivos propostos no manual da linhagem Agross (s.d.) para peso e ganho de peso das aves (machos e fêmeas) nas diferentes fases de criação foram aplicados em simulação matemática nos modelos elaborados para o cálculo da exigência de energia. Os resultados foram comparados ao consumo de EM preconizado pelo manual da marca comercial AgRoss 308.

\section{Resultados e Discussão}

Constam na Tabela 1 os valores de energia metabolizável ingerida (IEM), energia retida(ER) e produção de calor (PC) e a exigência de energia metabolizável para mantença (EMm) de frangos de corte submetidos a diferentes temperaturas $(13,23$ e $32^{\circ} \mathrm{C}$ ) e níveis de alimentação (ad libitum, 70, 50 e 30\% do ad libitum). Observou-se redução da IEM, da ER e do PC conforme o nível de alimentação decresceu. Blaxter (1989) comentou que o aumento da temperatura promove diminuição na ingestão de energia metabolizável e, conseqüentemente, na produção de calor, em razão do menor consumo de ração. Houve retenção negativa de energia nas aves submetidas à temperatura quente $\left(32^{\circ} \mathrm{C}\right)$ e ao menor nível de alimentação (30\% do consumo ad libitum). Entretanto, os valores de EMm obtidos demonstram que as exigências de energia para mantença das aves não foram alteradas pelo nível de alimentação, sendo, aproximadamente, de 137, 142, 129 e $132 \mathrm{kcal} / \mathrm{kg}^{0,75} /$ dia para os níveis de alimentação ad libitum, 70, 50 e 30\% do ad libitum, respectivamente.

Para as temperaturas de 13,23 e $32^{\circ} \mathrm{C}$, as exigências de EMm médias foram, respectivamente, 159,4; 116,2 e $128,7 \mathrm{kcal} / \mathrm{kg}^{0,75} / \mathrm{dia}$. Esses valores se aproximam muito da faixa de exigência de EMm (111,4 a $\left.143 \mathrm{kcal} / \mathrm{kg}^{0,75} / \mathrm{dia}\right)$ determinada por Nieto et al. (1995) para frangos de corte machos.

Tabela 1 - Médias e desvios-padrão obtidos para ingestão de energia metabolizável (IEM), energia retida no corpo (ER), produção de calor (PC) e exigência de EMm (EMm) para diferentes temperaturas e níveis de alimentação

Table 1 - Means and standard deviations of metabolizable energy intake (IEM), body energy retained (ER), heat production (PC), and MEm requirement (EMm), for different temperatures and feed intake levels

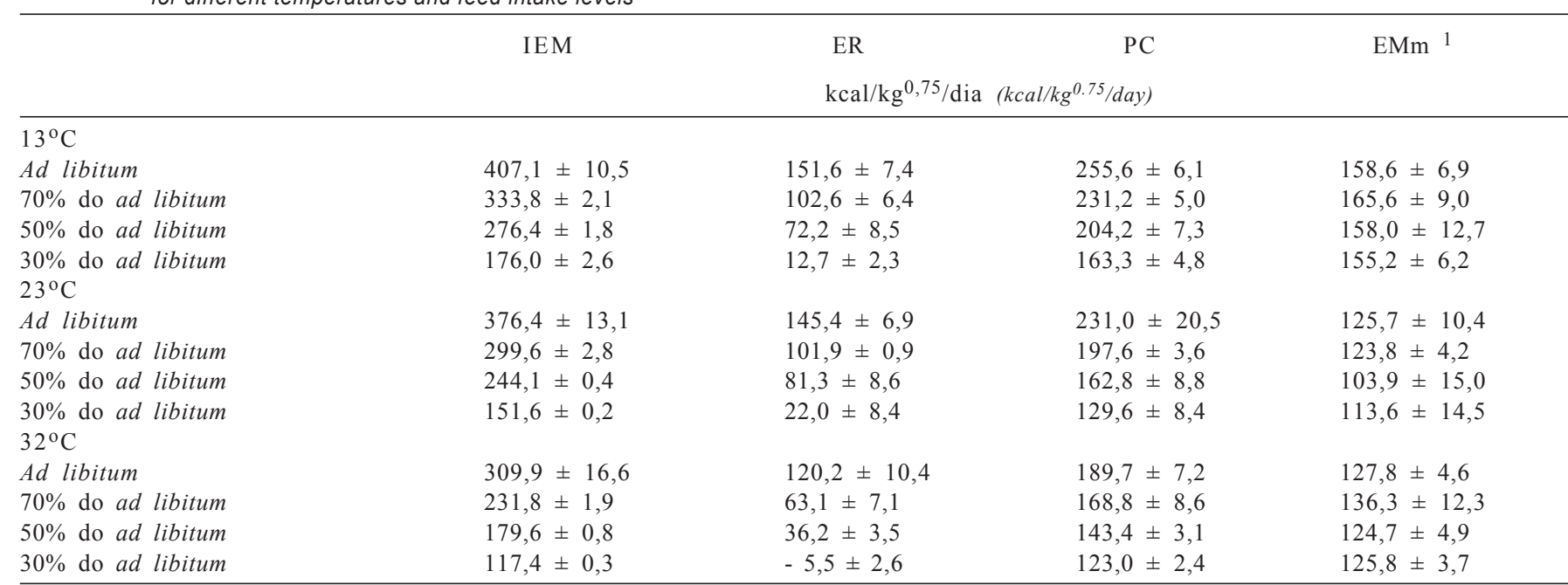

${ }^{1} \mathrm{EMm}=\mathrm{IEM}-(\mathrm{ER} / \mathrm{k})$, em que $\mathrm{k}=$ eficiência de utilização da energia para retenção corporal $\left(61,58\right.$ e $66 \%$ para 13,23 e $32^{\circ} \mathrm{C}$, respectivamente $($ Longo, 2000$)$.

${ }^{1} \mathrm{EMm}=I E M-(E R / \mathrm{k})$, where $\mathrm{k}=$ efficiency of metabolizable energy utilization for body retention $\left(61,58\right.$, and $66 \%$ to 13,23 , and $32^{\circ} \mathrm{C}$, respectively, Longo, 2000$)$. 
Observou-se efeito quadrático da temperatura $(\mathrm{P} \leq 0,05)$ sobre a EMm (Figura 1), o que promoveu decréscimo da exigência de mantença até $25,4^{\circ} \mathrm{C}$ e, a partir dessa temperatura, aumento, corroborando com os relatos de Leeson \& Summers (1997). Hurwitz et al. (1980) demonstraram que o efeito da temperatura sobre a exigência de energia não élinear e verificaram diminuição na exigência de mantença até $27^{\circ} \mathrm{C}$, seguida de um aumento até $34^{\circ} \mathrm{C}$. Esse efeito demonstra a maior necessidade de energia pelas aves submetidas a temperaturas acima ou abaixo da termoneutralidade; sob altas temperaturas, necessitam alterar seu metabolismo para dissipar calor e, sob temperaturas inferiores, aumentam a necessidade de produção de calor. Esses processos envolvem gastos de energia para manutenção da homeostase corporal.

Rabello etal. (2005) também observaram efeito quadrático da temperatura sobre a EMm $(\mathrm{EMm}=191,21-8,15 . \mathrm{T}+$ $0,16 . \mathrm{T}^{2} ; \mathrm{kcal} / \mathrm{kg}^{0,75} / \mathrm{dia}$ ) quando alojou reprodutoras pesadas em gaiolas.

O efeito quadrático da temperatura sobre a exigência de mantença neste trabalho difere dos encontrados por outros autores que relataram efeito linear da temperatura sobre as exigências de energia para mantença das aves (Sakomura, et al., 1993a, b; NRC, 1994; Sakomura et al., 2003, 2005).

A maioria dos modelos de predição das exigências de energia para poedeiras e matrizes pesadas destaca um efeito linear da temperatura sobre as exigências de mantença. Contudo, a relação linear deve ser considerada para as temperaturas próximas à zona de termoneutralidade (Sakomura, 1998).

Nesse contexto, a introdução de coeficientes que permitam relacionar a temperatura ambiente à exigência de EMm e, conseqüentemente, corrigir seus efeitos, possibilita melhores ajustes em equações de predição (Pesti et al., 1992).

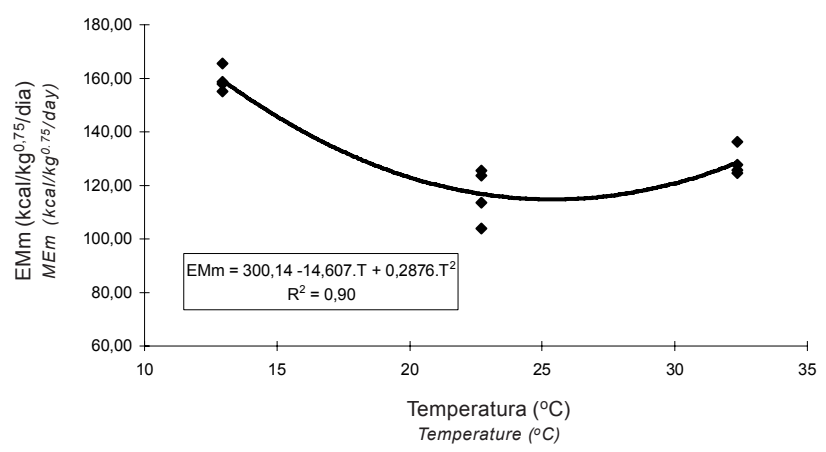

Figura 1 - Efeito da temperatura ambiente sobre a exigência de energia metabolizável para mantença. EMm (kcal/ $\left.\mathrm{kg}^{0,75} / \mathrm{dia}\right)=\mathrm{P}^{0,75} \cdot\left(300,14-14,607 . \mathrm{T}+0,2876 . \mathrm{T}^{2}\right)$, $R^{2}=0,90$, em que $P$ é o peso corporal em $\mathrm{kg} \mathrm{e} T$, a temperatura em ${ }^{\circ} \mathrm{C}$.

Figure 1 - Effect of environment temperature on the metabolizable energy requirements for maintenance. $M E m\left(\mathrm{kcal} / \mathrm{kg}^{0.75} /\right.$ day $)=W^{0.75}$. $\left(300.14-14.607 T+0.2876 T^{2}\right), R^{2}=0.90$, where $W$ is body weight $(\mathrm{kg})$ and $T$, temperature $(C)$.
Na Tabela 2 são apresentados os dados de peso corporal (P) e energia bruta corporal (EBc), observando-se maior crescimento corporal dos frangos machos em relação às fêmeas, conforme relatado também por Leeson \& Summers (1980) e Havenstein et al. (1994).

As exigências para ganho de peso foram determinadas para as três fases de criação ( 1 a 21, 22 a 42 e 43 a 56 dias de idade), acompanhando os aspectos fisiológicos do crescimento de frangos de corte.

Utilizando-se regressão linear entre os valores de energia bruta corporal em função do peso corporal (Tabela 2), determinou-se a exigência de energia líquida para ganho (ELganho) e, considerando-se a eficiência da utilização da energia $(k g=59 \%)$ em condições de termoneutralidade obtida por Longo (2000), estimou-se a exigência de energia metabolizável para ganho de peso (EMganho) para cada fase de criação (Tabela 3).

Observou-se crescente exigência de energia para o ganho de peso com o aumento da idade das aves (Tabela 3), de modo que os valores foram maiores para as fêmeas que para os machos nas fases inicial (1 a 21 dias) e final (43 a 56 dias). Estes resultados indicam maior exigência das fêmeas para deposição de um grama de peso corporal, justificando a adoção de um programa de alimentação diferente.

A diferença encontrada na fase final para machos e fêmeas pode estar relacionada à menor eficiência de crescimento, associada à maior taxa de deposição de gordura nas fêmeas nesse período. Longo (2000), em estudos sobre curvas de crescimento e deposição de proteína e gordura de frangos de corte de ambos os sexos, evidenciou que as diferenças nessas características de crescimento de fêmeas na fase final promovem alterações nas exigências energéticas para ganho de peso.

No modelo descrito por Hurwitz et al. (1978), as exigências para ganho de peso são iguais para ambos os sexos, ressaltando-se que a diferença nas exigências totais é decorrente da diferença na exigência de mantença.

Scott et al. (1982) comentaram que comparações entre as exigências de energia para ganho de peso em aves devem ser feitas com cautela, uma vez que, entre linhagens e sexo, ocorrem variações na composição corporal, na taxa de crescimento e, conseqüentemente, nas deposições de proteína e gordura corporal.

A partir dos valores de exigências de energia de mantença e ganho de peso, foram elaborados modelos de predição para as exigências diárias de EM para frangos de corte.

Os modelos foram determinados para cada fase de criação e sexo, considerando-se o peso corporal das aves $(\mathrm{P})$, em kg, o ganho de peso total diário (G), em gramas, e ainda a temperatura ambiente $(\mathrm{T}), \mathrm{em}{ }^{\circ} \mathrm{C}$ : 
Tabela 2 - Peso corporal e energia bruta corporal (EBc) para frangos de corte machos e fêmeas de 7 a 56 dias de idade Table 2 - Body weight and body gross energy $(E B C)$ for male and female broilers from 7 to 56 days old

\begin{tabular}{|c|c|c|c|c|}
\hline \multirow[t]{2}{*}{$\begin{array}{l}\text { Idade (dias) } \\
\text { Age (days) }\end{array}$} & \multicolumn{2}{|c|}{$\begin{array}{l}\text { Peso corporal }(\mathrm{g}) \\
\text { Body weight }(\mathrm{g})\end{array}$} & \multicolumn{2}{|c|}{$\begin{array}{c}\text { Energia bruta corporal (kcal) } \\
\text { Body gross energy (kcal) }\end{array}$} \\
\hline & Macho (Male) & Fêmea (Female) & Macho (Male) & Fêmea (Female) \\
\hline 7 & $134 \pm 5,5$ & $128 \pm 4,3$ & $112 \pm 2,6$ & $109 \pm 2,0$ \\
\hline 14 & $362 \pm 5,9$ & $340 \pm 11,9$ & $671 \pm 24,1$ & $692 \pm 49,8$ \\
\hline 21 & $700 \pm 30,5$ & $654 \pm 16,6$ & $1359 \pm 73,8$ & $1350 \pm 75,0$ \\
\hline 28 & $1181 \pm 39,8$ & $1050 \pm 26,0$ & $2762 \pm 184,2$ & $2451 \pm 215,3$ \\
\hline 35 & $1712 \pm 49,5$ & $1534 \pm 44,2$ & $4328 \pm 237,8$ & $3828 \pm 312,9$ \\
\hline 42 & $2320 \pm 45,7$ & $1968 \pm 44,7$ & $5319 \pm 432,7$ & $4322 \pm 190,2$ \\
\hline 49 & $2885 \pm 47,7$ & $2358 \pm 85,2$ & $6978 \pm 583,1$ & $5715 \pm 731,3$ \\
\hline 56 & $3503 \pm 34,7$ & $2745 \pm 95,5$ & $8475 \pm 1097,9$ & $7457 \pm 744,8$ \\
\hline
\end{tabular}

Tabela 3 - Equações de regressão da energia bruta corporal (EBc) em função do peso (P) e exigências de energias líquida (ELganho) e metabolizável (EMganho) para ganho de peso de frangos de corte Ross machos e fêmeas nas diferentes fases de criação

Table 3 - Regression equations of body gross energy $(E B C)$ as function of weight $(P)$ and requirements of net energy (ELganho) and metabolizable energy (EMganho) for weight gain of male and female broilers at different ages

\begin{tabular}{|c|c|c|c|c|}
\hline \multirow[t]{2}{*}{$\begin{array}{l}\text { Sexo } \\
\text { Sex }\end{array}$} & \multirow[t]{2}{*}{$\begin{array}{l}\text { Equação de regressão* } \\
\text { Regression equations* }\end{array}$} & \multirow[t]{2}{*}{$\mathrm{r}^{2}$} & \multicolumn{2}{|c|}{$\begin{array}{l}\text { Exigências para ganho de peso } \\
\text { Requirements for weight gain }\end{array}$} \\
\hline & & & $\begin{array}{c}\text { Líquida }(\mathrm{kcal} / \mathrm{g}) \\
\mathrm{Net}\end{array}$ & $\begin{array}{c}\text { Metabolizável }(\mathrm{kcal} / \mathrm{g}) \\
\text { Metabolizable }\end{array}$ \\
\hline \multicolumn{5}{|c|}{1 a 21 dias (1 to 21 days) } \\
\hline Macho (male) & $\mathrm{EBc}=-158,69+2,190 . P$ & 0,99 & 2,190 & 3,718 \\
\hline Fêmea (female) & $\mathrm{EBc}=-158,82+2,341 . P$ & 0,99 & 2,341 & 3,975 \\
\hline Macho (male) & $\mathrm{EBc}=-221,89+2,479 . P$ & 0,98 & 2,479 & 4,209 \\
\hline Fêmea (female) & $\mathrm{EBc}=-22,79+2,316 . P$ & 0,97 & 2,316 & 3,932 \\
\hline \multicolumn{5}{|c|}{43 a 56 dias (43 to 56 days) } \\
\hline Macho (male) & $\mathrm{EBc}=-788,54+2,657 . P$ & 0,89 & 2,657 & 4,511 \\
\hline Fêmea (female) & $\mathrm{EBc}=-3945,36+4,148 . P$ & 0,97 & 4,148 & 7,042 \\
\hline
\end{tabular}

* Equações de regressão deferiram significativamente de zero $(P \leq 0,05)$.

EMganho=ELganho/0,59 (coeficiente de regressão de $E R=-66,1105+0,5898$. IEM, $r^{2}=0,96$; Longo, 2000)

${ }^{*}$ Regression equations were significantly different from zero $(P \leq 0.05)$.

MEgain= NEgain/0.59 (coefficient of regression of RE $=-66.1105+0.5898$ IEM, $r^{2}=0.96 ;$ Longo, 2000).

1 a 21 dias: machos: $\mathrm{EM}(\mathrm{kcal} / \mathrm{ave} / \mathrm{dia})=\mathrm{P}^{0,75} \cdot(300,14-$ 14,61.T+0,2876. $\left.\mathrm{T}^{2}\right)+3,72 . \mathrm{G}$; fêmeas: $\mathrm{EM}(\mathrm{kcal} / \mathrm{ave} / \mathrm{dia})=$ $\mathrm{P}^{0,75} \cdot\left(300,14-14,61 \cdot \mathrm{T}+0,2876 \cdot \mathrm{T}^{2}\right)+3,98 \cdot \mathrm{G}$

22 a 42 dias: machos: $\mathrm{EM}(\mathrm{kcal} / \mathrm{ave} / \mathrm{dia})=\mathrm{P}^{0,75} \cdot(300,14-$ 14,61.T+0,2876. $\left.\mathrm{T}^{2}\right)+4,21 . \mathrm{G}$; fêmeas: $\mathrm{EM}(\mathrm{kcal} / \mathrm{ave} / \mathrm{dia})=$ $\mathrm{P}^{0,75} \cdot\left(300,14-14,61 . \mathrm{T}+0,2876 \cdot \mathrm{T}^{2}\right)+3,93 . \mathrm{G}$

43 a 56 dias: machos: $\mathrm{EM}(\mathrm{kcal} / \mathrm{ave} / \mathrm{dia})=\mathrm{P}^{0,75} \cdot(300,14-$ 14,61.T+0,2876. $\left.\mathrm{T}^{2}\right)+4,51 . \mathrm{G}$; fêmeas: $\mathrm{EM}(\mathrm{kcal} / \mathrm{ave} / \mathrm{dia})=$ $\mathrm{P}^{0,75} \cdot\left(300,14-14,61 . \mathrm{T}+0,2876 . \mathrm{T}^{2}\right)+7,04 . \mathrm{G}$

A partir dos dados de peso e ganho de peso preconizados pelo manual e de temperatura ambiente (Tabela 4) aplicados nos modelos elaborados, são apresentados nas Tabelas 5 e 6 os resultados dos consumos semanal e total de energia metabolizável para mantença e ganho de peso total e consumo de energia metabolizável recomendado pelo manual da linhagem de frangos de corte machos e fêmeas AgRoss 308 de 1 a 56 dias de idade.
Verificou-se aumento nas exigências de energia metabolizável (kcal/dia) de machos e fêmeas AgRoss 308 conforme aumentou a idade até 49 dias (Tabelas 5 e 6), ocorrendo decréscimo no período de 50 a 56 dias na exigência total, em decorrência da menor exigência para ganho com a diminuição na taxa de ganho de peso ( $\mathrm{g} /$ dia). Portanto, o aumento da exigência de mantença nesse período é proporcional ao do peso metabólico da ave. No fracionamento das exigências para mantença e ganho, observou-se proporção igual de utilização da energia para machos e fêmeas, sendo $36 \%$ para mantença e $64 \%$ para ganho de peso.

Comparando-se as exigências determinadas pelo modelo e a preconizada pelo manual da linhagem, constatou-se pelo modelo maior necessidade de energia no período de 1 a 49 dias e menor somente nos períodos de 50 a 56 dias (para machos) e de 36 a 42 dias (para as fêmeas). A equação considera uma queda na taxa de ganho a partir dessas fases 
Tabela 4 - Peso vivo médio (P), ganho de peso (GP) e temperaturas (T) semanais considerados para aplicação nos modelos de predição

Table 4 - $\quad$ Means of weekly body weight $(P)$, weight gain $(G P)$ and temperature $(T)$ considered in the prediction models

\begin{tabular}{|c|c|c|c|c|c|}
\hline \multirow[t]{2}{*}{$\begin{array}{l}\text { Idade (dias) } \\
\text { Age (days) }\end{array}$} & \multirow[t]{2}{*}{$\begin{array}{l}\mathrm{T}\left({ }^{\circ} \mathrm{C}\right) \\
T\left({ }^{\circ} \mathrm{C}\right)\end{array}$} & \multicolumn{2}{|c|}{$\begin{array}{l}P(\mathrm{~kg}) \\
P(\mathrm{~kg})\end{array}$} & \multicolumn{2}{|c|}{$\begin{array}{l}\text { GP }(\mathrm{g} / \mathrm{dia}) \\
G P \\
\text { G }\end{array}$} \\
\hline & & Macho (Male) & Fêmea (Female) & Macho (Male) & Fêmea (Female) \\
\hline 1 a 7 & 28,0 & 0,12 & 0,12 & 21,86 & 20,86 \\
\hline 8 a 14 & 27,6 & 0,34 & 0,32 & 41,29 & 38,00 \\
\hline 15 a 21 & 28,4 & 0,69 & 0,63 & 58,86 & 51,57 \\
\hline 22 a 28 & 26,7 & 1,15 & 1,03 & 72,43 & 60,57 \\
\hline 29 a 35 & 27,6 & 1,68 & 1,46 & 78,71 & 62,14 \\
\hline 36 a 42 & 27,8 & 2,23 & 1,88 & 78,57 & 58,29 \\
\hline 43 a 49 & 26,6 & 2,78 & 2,27 & 78,29 & 54,43 \\
\hline 50 a 56 & 25,7 & 3,28 & 2,61 & 66,43 & 43,29 \\
\hline
\end{tabular}

Tabela 5 - Consumo de energia metabolizável (EM) determinado pelos modelos de predição, fracionamento para mantença e ganho e consumo de EM preconizado no manual para frangos de corte machos Ross

Table 5 - Metabolizable energy (ME) intake determined by the prediction models, ME for maintenance and weight gain, and ME intake predicted by the manual recommendations for male Ross broilers

\begin{tabular}{|c|c|c|c|c|}
\hline $\begin{array}{l}\text { Idade (dias) } \\
\text { Age (days) }\end{array}$ & $\begin{array}{l}\text { EM modelos }{ }^{1} \\
M E \text { models }^{1}\end{array}$ & $\begin{array}{l}\mathrm{EM} \text { mantença } \\
M E \text { maintenance }\end{array}$ & $\begin{array}{l}\text { EM ganho } \\
M E \text { gain }\end{array}$ & $\begin{array}{l}\text { EM manual } \\
M E \text { handbook }\end{array}$ \\
\hline & \multicolumn{4}{|c|}{$\begin{array}{c}\text { (kcal/dia) } \\
(\text { kcal/day })\end{array}$} \\
\hline 1 a 7 & 103,81 & 22,50 & 81,31 & 61,92 \\
\hline 8 a 14 & 204,25 & 50,67 & 153,6 & 166,4 \\
\hline 15 a 21 & 306,84 & 87,89 & 218,9 & 283,5 \\
\hline 22 a 28 & 432,05 & 127,13 & 304,9 & 375,1 \\
\hline 29 a 35 & 501,99 & 170,61 & 331,4 & 462,6 \\
\hline 36 a 42 & 542,43 & 211,64 & 330,8 & 533,0 \\
\hline 43 a 49 & 600,24 & 247,17 & 353,1 & 584,6 \\
\hline 50 a 56 & 579,09 & 279,50 & 299,6 & 633,9 \\
\hline Total (kcal) & 22895 & 8380 & 14515 & 21708 \\
\hline
\end{tabular}

11 a 21 dias de idade: $E M(\mathrm{kcal} / \mathrm{ave} / \mathrm{dia})=\mathrm{P}^{0,75} \cdot\left(300,14-14,61 \cdot \mathrm{T}+0,2876 \cdot \mathrm{T}^{2}\right)+3,72 \cdot \mathrm{G}, 22$ a 42 dias de idade: $\mathrm{EM}(\mathrm{kcal} / \mathrm{ave} / \mathrm{dia})=$ $\mathrm{P}^{0,75} \cdot\left(300,14-14,61 \cdot \mathrm{T}+0,2876 \cdot \mathrm{T}^{2}\right)+4,21 \cdot \mathrm{G}, 43$ a 56 dias de idade: $\mathrm{EM}(\mathrm{kcal} / \mathrm{ave} / \mathrm{dia})=\mathrm{P}^{0,75} \cdot\left(300,14-14,61 \cdot \mathrm{T}+0,2876 \cdot \mathrm{T}^{2}\right)+4,51 . \mathrm{G}$ 11 to 21 days old: $M E(k c a l / b i r d / d a y)=W^{0} \cdot 75 .\left(300.14-14 \cdot 61 \cdot T+0.2876 \cdot T^{2}\right)+3.72 G, 22$ to 42 days old: $M E(k c a l / b i r d / d a y)=W^{0.75} \cdot\left(300 \cdot 14-14 \cdot 61 \cdot T+0.2876 \cdot T^{2}\right)+$ 4.21G, 43 to 56 days old: $M E(k c a l / /$ bird/day $)=W^{0.75} \cdot\left(300.14-14.61 . T+0.2876 . T^{2}\right)+4.51 G$

Tabela 6 - Consumo de energia metabolizável (EM) determinado pelos modelos de predição, fracionamento para mantença e ganho e consumo de EM preconizado no manual para frangos de corte Ross fêmeas

Table 6 - Metabolizable energy (ME) intake determined by the prediction models, ME for maintenance and weight gain, and ME intake predicted by the manual recommendations for female Ross broilers

\begin{tabular}{|c|c|c|c|c|}
\hline $\begin{array}{l}\text { Idade (dias) } \\
\text { Age (days) }\end{array}$ & $\begin{array}{l}\text { EM modelos }{ }^{1} \\
M E \text { models }^{1}\end{array}$ & $\begin{array}{l}\text { EM mantença } \\
\text { ME maintenance }\end{array}$ & $\begin{array}{l}\text { EM ganho } \\
M E \text { gain }\end{array}$ & $\begin{array}{l}\text { EM manual } \\
M E \text { handbook }\end{array}$ \\
\hline & \multicolumn{4}{|c|}{$\begin{array}{c}\text { (kcal/dia) } \\
(\text { kcal/day })\end{array}$} \\
\hline 1 a 7 & 105,1 & 22,0 & 83,0 & 60,6 \\
\hline 8 a 14 & 199,9 & 48,6 & 151,2 & 157,4 \\
\hline 15 a 21 & 287,9 & 82,6 & 205,3 & 255,3 \\
\hline 22 a 28 & 354,9 & 116,9 & 238,0 & 327,3 \\
\hline 29 a 35 & 397,7 & 153,5 & 244,2 & 389,3 \\
\hline 36 a 42 & 415,2 & 186,2 & 229,1 & 432,5 \\
\hline 43 a 49 & 595,8 & 212,7 & 383,2 & 456,1 \\
\hline 50 a 56 & 540,3 & 235,6 & 304,7 & 476,1 \\
\hline Total (kcal) & 20277 & 7406 & 12871 & 17882 \\
\hline
\end{tabular}

11 a 21 dias de idade: $E M(k c a l / a v e / d i a)=P^{0,75} \cdot\left(300,14-14,61 \cdot T+0,2876 \cdot T^{2}\right)+3,72 \cdot G, 22$ a 42 dias de idade: $E M(k c a l / a v e / d i a)=$ $\mathrm{P}^{0,75} \cdot\left(300,14-14,61 \cdot \mathrm{T}+0,2876 \cdot \mathrm{T}^{2}\right)+4,21 \cdot \mathrm{G}, 43$ a 56 dias de idade: $\mathrm{EM}(\mathrm{kcal} / \mathrm{ave} / \mathrm{dia})=\mathrm{P}^{0,75} \cdot\left(300,14-14,61 \cdot \mathrm{T}+0,2876 \cdot \mathrm{T}^{2}\right)+4,51 \cdot \mathrm{G}$ 11 to 21 days old: $M E(\mathrm{kcal} / / \mathrm{bird} /$ day $)=W^{0.75} \cdot\left(300.14-14.61 . T+0.2876 . T^{2}\right)+3.72 \mathrm{G}, 22$ to 42 days old: $M E(\mathrm{kcal} / \mathrm{bird} /$ day $)=W^{0} \cdot 75 \cdot\left(300.14-14.61 . T+0.2876 . T^{2}\right)+$ 4.21G, 43 to 56 days old: $M E(k c a l / / b i r d / d a y)=W^{0.75} \cdot\left(300.14-14.61 . T+0.2876 . T^{2}\right)+4.51 G$ 
(Tabela 4). Para as exigências de energia total no período de 1 a 56 dias, verificou-se maior necessidade de energia metabolizável determinada pela equação, demonstrando a importância dos fatores utilizados na equação para correção das exigências energéticas. A diferença entre a exigência total de energia determinada pela equação e a preconizada no manual foi maior para fêmeas, indicando a necessidade de se determinarem as exigências de energia metabolizável para mantença para os diferentes sexos, como descrito por Hurwitz et al. (1978).

As exigências de energia para as fêmeas foram sempre inferiores à dos machos, principalmente em razão do menor peso e do ganho de peso, concordando com valores descritos por Shalev \& Pasternak (1998).

Outros fatores que influenciam as exigências de energia, como umidade, movimento do ar, densidade das aves (Zhang \& Coon, 1994) e empenamento (Peguri \& Coon, 1993 ) não foram estudados e incorporados aos modelos de predição neste estudo, o que pode ter influenciado a predição mais exata das exigências de energia, evidenciando que maiores ajustes ainda devem ser estudados visando à determinação de exigências para aves.

\section{Conclusões}

Os modelos elaborados são precisos para predizer as exigências de energia metabolizável para frangos de corte da marca comercial AgRoss 308 em diferentes fases de criação.

\section{Agradecimento}

À Fundação de Amparo à Pesquisa do Estado de São Paulo (FAPESP), pelo suporte financeiro.

\section{Literatura Citada}

ALBINO, L.F.T.; NASCIMENTO, A.H.; VALÉRIO, S.R. Níveis de energia da dieta e da temperatura ambiente sobre a composição da carcaça em frangos (músculo e gordura). In: CONFERÊNCIA APINCO DE CIÊNCIA E TECNOLOGIA AVÍCOLAS - FACTA, 2000, Campinas. Anais... Campinas: Fundação Apinco de Ciências e Tecnologia Avícolas, 2000. p.62-79.

AGROSS, s.d. Manual de manejo de frango de corte. Agroceres Ross.

BLAXTER, K. Energy metabolism in animals and man. Cambridge: Press Syndicate of the University of Cambridge, 1989. p.23-65.

EMMANS, G.C. Growth, body composition and feed intake. World's Poultry Science Journal, v.43, p.208-227, 1986.

HAVENSTAIN, G.B.; FERKET, P.R.; SCHEIDLER, S.E. et al. Growth, livability and feed conversion when "typical"1957 and 1991 broiler diets. Poultry Science, v.73, p.1785-1794, 1994.

HURWITZ, S.; PLAVINIK, I.; BENGAL, I. et al. The amino acid requirements of growing turkeys. 1- Model construction and parameter estimation. Poultry Science, v.62, p.2208$2217,1983$.

HURWITZ, S.; WEISELBERG, M.; EISNER, U.T. The energy requirements and performance of growing chickens and turkeys as affected by environmental temperature. Poultry Science, v.59, p.2121-2128, 1980 .

HURWITZ, S.; SKLAN, D.; BARTOV, I. New formal approaches to determination of energy and amino acid requirements of chickens. Poultry Science, v.57, p.197-205, 1978

LEESON, S.; SUMMERS, J.D. Commercial poultry nutrition. 2.ed. Guelph: University Books, 1997. 355p.

LEESON, S.; SUMMERS, J.D. Production and carcass characteristics of the broiler chicken. Poultry Science, v.59, p.786-798, 1980.

LONGO, F.A. Estudo do metabolismo energético e do crescimento em frangos de corte. Jaboticabal: FCAV-UNESP, Campus de Jaboticabal: Universidade Estadual Paulista, 2000. 76p. Dissertação (Mestrado em Zootecnia) - Universidade Estadual Paulista, 2000.

NIETO, R.; PRIETO, C.; FERNANDEZ-FIGARES, I. et al. Effect of dietary protein quality on energy metabolism in growing chickens. British Journal Nutrition, v.74, p.163-172, 1995.

NATIONAL RESEARCH COUNCIL - NRC. Nutrient requirements of poultry. 9.ed. Washington, D.C.: National Academy of Sciences, 1994. 155p.

PEGURI, A.; COON, C.N. Development and evaluation of prediction equations for metabolizable energy and true metabolizable energy intake for Dekalb XL-Link White Leghorn hen. In: MINNESOTA NUTRITION CONFERENCE AND DEGUSSA TECHNOLOGY SYMPOSIUM, 49., 1988, Bloming. Proceedings... Bloming: 1988. p.199-211.

PESTI, G.M.; DORFMAN, J.H.; GONZALES, M.J. Comparison of equations for predicting the metabolizable energy intake of laying pullets. British Poultry Science, v.33, p.553-559, 1992.

RABELLO, C.B.V.; SAKOMURA, N.K.; LONGO, F.A. et al. Temperatura ambiente e do sistema de criação sobre as exigências de energia metabolizável para mantença de aves reprodutoras pesadas. Revista Brasileira de Zootecnia, v.33, n.2, p.382$390,2005$.

SAKOMURA, N.K. Influência da temperatura ambiente sobre a exigência nutricional de aves (frangos de corte, matrizes e galinhas poedeiras). In: CONFERËNCIA APINCO DE CIENCIA E TECNOLOGIA - FACTA, 1998, Campinas. Anais... Campinas: Fundação Apinco de Ciências e Tecnologia Avícolas, 1998. p.267-293.

SAKOMURA, N.K.; ROSTAGNO, H.S.; TORRES, R.A. et al. Efeito da temperatura sobre o consumo de ração e de energia para matrizes pesadas. Revista da Sociedade Brasileira de Zootecnia, v.22, n.5, p.707-714, 1993a.

SAKOMURA, N.K.; ROSTAGNO, H.S.; SOARES, P.R. et al. Determinação das equações de predição da exigência nutricional de energia para matrizes e poedeiras. Revista da Sociedade Brasileira de Zootecnia, v.22, n.5, p.723-731, 1993b.

SAKOMURA, N.K.; SILVA, R.; COUTO, H.P. et al. Modeling metabolizable energy utilization in broiler breeder pullets. Poultry Science, v. 82, p.419-427, 2003.

SAKOMURA, N.K.; BASAGLIA, R.; FORTES, C.M.L.S. et al. Modelos para determinar as exigências de energia metabolizável para poedeiras. Revista Brasileira de Zootecnia, v.34, n.2, p. 507-515, 2005.

SHALEV, B.A.; PASTERNAK, H. The relative energy requirements of male vs female broilers and turkeys. Poultry Science, v.77, p. 859-863, 1998.

SCOTT, M.L.; NESHEIM, M.C.; YOUNG, R.J. Nutrition of the chicken. 3.ed. Ithaca: M.L. Scott \& Association, 1982. 562p. STATISTICA, 1996. Version Release 5.1. for windows. 